\title{
Simposio Ibero-Latinoamericano de Terminología Morfológica. Características Generales
}

\author{
Ibero-Latin-American Symposium of Morphological Terminology. General Characteristics
}

\author{
"Rolando Cruz Gutiérrez; **Alberto Rodríguez Torres; **** José Carlos Prates; \\ ***** Ricardo Jorge Losardo \& ${ }^{* * * * * *}$ Nadir Eunice Valverde Barbato de Prates
}

CRUZ, G. R.; RODRÍGUEZ, T. A.; PRATES, J. C.; LOSARDO, R. J. \& PRATES, N. E. V. B. Simposio Ibero-latinoamericano de Terminología Morfológica. Características Generales. Int. J. Morphol., 28(2):643-646, 2010.

RESUMEN:Se describen las principales características de SILAT como programa científico y educativo multinacional, que sirve de instrumento para analizar y solucionar los problemas de la terminología médica morfológica en los países de habla hispana y portuguesa de América. Se detallan antecedentes y creación, miembros, fines y funciones, medios, actividades, estructura organizativa, consejo directivo, relaciones con otros organismos y publicaciones.

PALABRAS CLAVE: Simposio; Ibero-latinoamericano; Terminología; Morfología.

Las actividades internacionales relacionadas con las terminologías morfológicas tienen necesidad de contar con la cooperación de los profesores ibero-latinoamericanos con el fin de lograr cabalmente sus objetivos científicos y educativos en el área de la salud.

Los profesores e investigadores universitarios, de ciencias morfológicas ibero-latinoamericanos han tenido una progresiva toma de conciencia de su identidad comunitaria y de su valiosa ayuda en aquel proyecto internacional.

Como resultado de ambas circunstancias, se ha impulsado la creación de esta entidad, el Simposio Ibero-latinoamericano de Terminología (SILAT) -anatómica, histológica y embriológica- que serviría como instrumento para la solución de problemas comunes y cuya participación será creciente en el desarrollo de las ciencias de la salud en la región y su aporte en el mundo.

A continuación describiremos las principales características de este organismo no gubernamental (ONG), de índole científico y educativo multinacional, señalando sus antecedentes, creación, miembros, fines, contribuciones financieras, estructura, reuniones, sedes, etc.

\section{ANTECEDENTES Y CREACIÓN}

Durante la realización del Simposio Ibero-latinoamericano de Terminología (SILAT) Anatómica e Histológica, organizado por la Asociación Panamericana de Anatomía (APA), en San José de Costa Rica, entre el 13 y 16 de abril de 2009, se realizó una reunión de trabajo donde se firmó un acta fundacional entre los distintos profesores de ciencias morfológicas de Latinoamérica y autoridades de la Asociación Panamericana de Anatomía (APA), con el que se inicia la creación de este grupo de trabajo, que se reunirá en el SILAT, a fin de fortalecer los vínculos científicos y educativos que unen a los países signatarios.

A esta primera reunión acudieron representantes de los siguientes países: Argentina, Brasil, Colombia, Costa Rica, Chile, Ecuador, Guatemala, Honduras, México, Nicaragua y Perú (Anexo 1).

A partir de la firma de aquella acta de Costa Rica, SILAT funciona como un programa creado y auspiciado por APA con el propósito de realizar reuniones ordinarias con sedes en los distintos países de la región, a fin de revi-

\footnotetext{
* Presidente de Asociación Panamericana de Anatomía, Miembro de FICAT, Facultad de Medicina, Universidad de Costa Rica (UCR), San José, Costa Rica.

** Presidente Honorario de Asociación Panamericana de Anatomía, Facultad de Medicina, Universidad de Chile, Santiago, Chile.

*** Presidente Honorario de Asociación Panamericana de Anatomía, Ex Miembro de FICAT, Universidade Federal de São Paulo (UNIFESP), São Paulo, Brasil.

${ }^{* * * * *}$ Presidente Honorario de Asociación Panamericana de Anatomía, Facultad de Medicina, Universidad del Salvador (USAL), Buenos Aires, Argentina.

${ }^{* * * * *}$ Secretaria General de Asociación Panamericana de Anatomía, Instituto de Ciencias Biomédicas (ICB), Universidade de São Paulo (USP), São Paulo, Brasil.
} 
CRUZ, G. R.; RODRÍGUEZ, T. A.; PRATES, J. C.; LOSARDO, R. J. \& PRATES, N. E. V. B. Simposio Ibero-latinoamericano de Terminología Morfológica. Características Generales. Int. J. Morphol., 28(2):643-646, 2010.

sar, estudiar, analizar, discutir, difundir y traducir regularmente la terminología morfológica.

\section{MIEMBROS}

Son miembros de SILAT los profesores e investigadores universitarios de los países ibero-latinoamericanos y actualmente están representados los siguientes países:Argentina, Brasil, Colombia, Costa Rica, Chile, Ecuador, Guatemala, Honduras, El Salvador, México, Nicaragua y Perú.

\section{FINES Y FUNCIONES}

1. Estimular y fomentar el conocimiento de la terminología internacional morfológica a través de simposios, seminarios, jornadas, cursos, mesas redondas, conferencias, publicaciones, en las universidades de los países de habla hispana y portuguesa.

2. Estudiar y analizar las traducciones española y portuguesa de la terminología internacional morfológica procedentes de la versión latina.

3. Promover capacitación y brindar adiestramiento -en esta materia- a los docentes e investigadores universitarios de esta región.

4. Efectuar y facilitar investigaciones en las universidades de los países ibero-latinoamericanos, con el objeto de analizar los problemas de las terminologías anatómica, histológica y/o embriológica y sus posibles soluciones.

5. Asesorar a los países miembros en el estudio e implementación de reformas en los programas tendientes a modernizar la enseñanza universitaria en el campo de la terminología internacional.

6. Sustentar, como finalidad primordial, la integración regional a la terminología internacional vigente.

\section{MEDIOS}

En la actualidad SILAT funciona como una actividad auspiciada por la Asociación Panamericana de Anatomía y la Universidad organizadora de turno.

Los gastos de organización y desarrollo de SILAT corren por cuenta del país miembro organizador del Simposio o reunión que corresponda.
Las donaciones recibidas de personas físicas o jurídicas y de entidades nacionales, regionales e internacionales serán utilizadas exclusivamente para la organización y desarrollo de las actividades de SILAT.

\section{ACTIVIDADES}

Las actividades de SILAT se concretan en los siguientes puntos:

1. Promoción: coordinar y promover los esfuerzos para la difusión y utilización de la terminología internacional en las universidades ibero-latinoamericanas.

2. Docencia: adiestramiento y capacitación de docentes en esta materia (en especial del sector público) a través de simposios, seminarios, jornadas, cursos, etc. Una función básica de SILAT es la formación de instructores con el fin de lograr un efecto multiplicador. Consiste en preparar cuadros técnicos, capaces de llevar a cabo -en el ámbito nacional- labores de adiestramiento en las áreas específicas (anatomía, embriología e histología) para las que han sido adiestrados.

3. Investigación: son estudios -tanto a nivel regional como nacional- que sirven de base para la búsqueda de un lenguaje científico común para toda la comunidad ibero-latinoamericana de las ciencias morfológicas. Además, se formulan propuestas de terminologías regionales y nacionales, que se ponen a consideración y discusión en el Comité de Expertos de SILAT.

4. Publicaciones: llevar a cabo una labor de documentación en el campo científico y educativo; y realizar una serie de publicaciones: a) informes de simposios, seminarios, jornadas, conferencias, mesas redondas; y b) folletos, boletines, cuadernillos y libros sobre terminología anatómica, histológica y embriológica.

5. Asesoría técnica: considera proyectos de asistencia y cooperación técnica en la materia. Se ofrece a las universidades ibero-latinoamericanas y organismos que lo deseen.

La primera reunión, fundacional y de trabajo, se celebró en San José de Costa Rica, entre el 13 y el 16 de abril de 2009, bajo la presidencia del Prof. Dr. Rolando Cruz Gutiérrez y bajo el título de I Simposio Ibero-latinoamericano de Terminología.

La segunda reunión tuvo lugar en Lima- Perú, entre el 15 y el 18 de septiembre de 2009, bajo la presidencia del Prof. Dr. Germán Molina Albornoz y bajo el título de II Simposio Ibero-latinoamericano de Terminología. 
La tercera reunión, se celebró nuevamente en San José de Costa Rica, entre el 26 y el 30 de abril de 2010, bajo la presidencia del Prof. Dr. Rolando Cruz Gutiérrez y bajo el título de III Simposio Ibero-latinoamericano de Terminología.

\section{ESTRUCTURA ORGANIZATIVA}

La autoridad superior de SILAT está constituida por un Consejo directivo permanente, integrado por los Presidentes Honorarios de la APA y los Ex - Presidentes de Simposios realizados; y queda a disposición para consultas y asesoramientos.

El Comité organizador local del Simposio está integrado por los representantes del país organizador. Dirige y es responsable de la organización del foro. Cada Simposio tiene su Presidente y es nombrado por los países asistentes en la última reunión previa al evento.

En la sede donde se realiza el Simposio se reúne el Comité de expertos formado por profesores e investigadores universitarios ibero-latinoamericanos dedicados a esta problemática. Difunden y estudian la terminología presentada por el Comité Federal sobre la Terminología Anatómica (FICAT) órgano especializado de la Federación Internacional de Asociaciones de Anatomía (IFAA)-. También la traducen, sugiriendo criterios para su adaptación al habla hispana y portuguesa. Asimismo, estudian los asuntos que el FICAT encomiende para la región. Finalmente, plantean a esa Federación las diferencias surgidas en el seno de los Simposios y que no hayan logrado consenso en su solución por parte del Comité de Expertos de SILAT.

\section{CONSEJO DIRECTIVO}

Integrado actualmente por los Prof. Dres. Rolando Cruz Gutiérrez, José Carlos Prates, Alberto Rodríguez Torres y Ricardo Jorge Losardo (Presidentes Honorarios de APA activos) y Germán Molina Albornoz (Ex - Presidente de SILAT).

Los futuros presidentes honorarios de APA y los Ex Presidentes de SILAT, se irán incorporando a este Consejo Directivo en forma automática.

Es el órgano ejecutivo y administrativo máximo de SILAT, que fija la política a seguir. Revisa y aprueba el programa científico del Comité organizador de cada evento. Tiene la finalidad de asegurar la continuidad de las sedes de los eventos y de las actividades durante el período entre dos eventos. También resuelve los asuntos urgentes.

\section{RELACIONES CON OTROS ORGANISMOS}

SILAT constituye un órgano técnico de consulta de la Asociación Panamericana de Anatomía (Nivel 2). A través de ésta se relaciona con las Sociedades Científicas Nacionales (Nivel 1) y con la Federación Internacional de Asociaciones de Anatomistas (Nivel 3).

Se plantea realizar convenios de cooperación técnica con universidades, escuelas, sociedades científicas y organismos nacionales o internacionales que procuren incentivar el progreso en esta área de las ciencias de la salud y de la biología.

\section{PUBLICACIONES}

Se publican los informes y actas de los simposios y reuniones para su distribución a los participantes de los países miembros.

SILAT publica en las siguientes revistas: Archivos Anatómicos de Costa Rica (suplemento de la Revista Médica de Costa Rica y Centroamérica); Internacional Journal of Morphology y Brazilian Journal of Morphological Sciences.

\section{Anexo 1. ACTA FUNDACIONAL DEL SIMPOSIO IBERO- LATINOAMERICANO DE TERMINOLOGÍA (SILAT). San José de Costa Rica, 15 de abril de 2009}

En la ciudad de San José de Costa Rica y dentro del marco del Simposio Ibero-latinoamericano de Terminología Anatómica e Histológica, organizado por la Asociación Panamericana de Anatomía, realizado entre los días 13 y 16 de abril de 2009, se reúnen por primera vez en el continente americano, profesores de anatomía, histología y embriología de habla hispana y portuguesa, todos ellos miembros de la Asociación Panamericana de Anatomía; con la coordinación del Presidente y de la Secretaría General y de 2 ex -Presidentes de dicha Asociación.

Tienen el objeto de revisar, estudiar, analizar, discutir y divulgar la Terminología Anatómica e Histológica de la FICAT (dependiente de la Federación Internacional de Anatomía) y la traducción al español realizada por la Sociedad Anatómica Española y la traducción al portugués de la Sociedad Brasileña de Anatomía; procurando también el conocimiento de dichas terminologías (latina, española y portuguesa) en nuestro continente. 
En esta primera reunión de trabajo se hace el aporte de los morfólogos de países latinoamericanos, con el fin de enviar comentarios y sugerencias sobre la lista -latina, española y portuguesa- de términos y la posibilidad de conciliar posiciones y evitar omisiones de la actual edición para la realización de la próxima; colaborando en la confección de la misma.

De esta manera, se representa la realidad de esta ciencia en el conjunto de países que aquí se encuentran, y con el compromiso de seguir llevando a cabo otras reuniones de trabajo en otras universidades, a fin de analizar anualmente la lista latina de términos anatómicos, histológicos y embriológicos, base de la terminología médica.

La participación de los concurrentes a estas reuniones de trabajo será certificada por la Asociación Panamericana de Anatomía y la Universidad organizadora del evento.

\section{SIGNATARIOS DELACTA DE SAN JOSÉ DE COSTA RICA}

Avila, Rodolfo Esteban; Barco Ríos, John; Becerril Montes, Adriana; Coello Cunto, Rafael; Coello Salguero, Rafael; Cruz Gutiérrez, Rolando (Presidente); Duque Parra, Jorge Eduardo; Enriquez Martínez, Ana María; Enríquez Paz, Juan Carlos; Franco, Roberto J.; Grillo Bustamante, Eduardo; Gutiérrez Vargas, Gabriela; Herrera Saint - Leu, Patricia Margarita; Leiva Orozco, Osman Albino; Letona González Augusto René; Losardo, Ricardo Jorge (Ex -Presidente); Masuko, Telma Sumic; Molina Albornoz, Vladimir Germán; Morataya Roldán, César Augusto; Pardo Angulo, Patricia Enriqueta; Rodríguez Torres, Alberto (Ex - Presidente); Samar, María Elena; Santos Reyes, Alda Clementina; Valverde Barbato de Prates, Nadir Eunice (Secretaria General); Villalobos Quesada, Lucrecia; Zamora Vargas, Luis Eduardo.

CRUZ, G. R.; RODRÍGUEZ, T. A.; PRATES, J. C.; LOSARDO, R. J. \& PRATES, N. E. V. B. Ibero-Latin-American Symposium of Morphological Terminology. General Characteristics. Int. J. Morphol., 28(2):637-642, 2010.

SUMMARY: It describes the main features of SILAT as a multinational scientific and educational program that serves as a tool to analyze and solve the morphological medical terminology problems in Spanish and Portuguese speaking countries of America. It treated history and creation, members, aims and functions, resources, activities, organizational structure, board, relations with other organizations and publications.

KEY WORDS: Symposium; Ibero-LatinAmerican; Morphology; Terminology.

\section{REFERENCIAS BIBLIOGRÁFICAS}

Cruz Gutiérrez, R.; Rodríguez Torres, A.; Prates, J. C.; Losardo, R. J. \& Valverde Barbato, N.: Simposios Ibero-latinoamericanos de Terminología. Anatomía, Histología y Embriología. Int. J. Morphol., 28(1):33740, 2010.

Losardo, R. J. Asociación Panamericana de Anatomía: Reseña histórica y normativas vigentes. Int. J. Morphol., 27(4):1345-52, 2009.
Dirección para correspondencia

Prof. Dr. Ricardo Jorge Losardo

Lavalle 1844 - Piso $5^{\circ}$ - Oficina 30

(1051) Ciudad de Buenos Aires ARGENTINA

Tel / Fax.: 0054-11-4372-4604

Email: ricardo.losardo@salvador.edu.ar

Recibido : 19-05-2010

Aceptado: $27-05-2010$ 